\title{
Modelling Spin Transport in Helical Fields: Derivation of an Effective Low-dimensional Hamiltonian
}

\author{
R. Gutierrez¹, E. Díaz², C. Gaull2,3 T. Brumme¹, F. Domínguez-Adame², and G. \\ Cuniberti $^{1}$ \\ ${ }^{1}$ Institute for Materials Science, Dresden University of Technology, 01062 Dresden, \\ Germany \\ ${ }^{2}$ GISC, Departamento de Física de Materiales, Universidad Complutense, E-28040 Madrid, \\ Spain \\ ${ }^{3}$ CEI Campus Moncloa, UCM-UPM, E-28040 Madrid, Spain
}

The Journal of Physical Chemistry C 177, 22276 (2013)

E-mail: 


\begin{abstract}
This study is devoted to a consistent derivation of an effective model Hamiltonian to describe spin transport along a helical pathway and in presence of spin-orbit interaction, the latter being induced by an external field with helical symmetry. It is found that a sizeable spin polarization of an unpolarized incoming state can be obtained without introducing phase breaking processes. For this, at least two energy levels per lattice site in the tight-binding representation are needed. Additionally, asymmetries in the effective electronic-coupling parameters as well as in the spin-orbit interaction strength must be present to achieve net polarization. For a fully symmetric system - in terms of electronic and spin-orbit couplings - no spin polarization is found. The model presented is quite general and is expected to be of interest for the treatment of spindependent effects in molecular scale systems with helical symmetry.
\end{abstract}

Keywords: charge transport, Green's functions, spin polarization, Landauer theory, curvilinear coordinates 


\section{Introduction}

Gaining control over the spin degree of freedom to transfer information lies at the very basis of spintronics. Up to date, the majority of existing spintronic devices are based on inorganic materials. However, an alternative route that exploits organic molecules would offer many advantages, including the chemical control of the the spin-dependent response of the system as well as the rather inexpensive synthesis of identical molecular-scale building blocks. Some work has been performed on organic molecules, suggesting them as spinvalves. 15 However, as a rule, the spin sensitivity of molecular based spintronics is rather related to the magnetic properties of the electrodes or of the used molecules, so that for non-magnetic molecular systems it is unlikely that strong spin-dependent effects will arise. Hence, the recent experimental demonstration $\sqrt{6 / 7}$ of spin selective effects in monolayers of double-stranded DNA oligomers as well as earlier works ${ }^{8}[15$ have triggered strong interest. As a working hypothesis, it was suggested that the observed spin selectivity may be related to the specific geometric structure of the involved molecular systems, namely their helical conformation. 7

On the theoretical side, some investigations based on minimal model approaches including the helical symmetry of the system have been published. In brief, two main lines can be identified up to now:

i. Studies based on scattering theory at the level of the Born approximation, $\frac{16117}{1 n c l u d i n g}$ spin-orbit coupling (SOC) derived from a helically shaped potential. This approach can be closely related to the experiments in Ref. 6, where the energies of the emitted electrons lie well above the energy of molecular orbitals of the DNA molecules and thus, the problem can be viewed as a scattering process in an external helical potential.

ii. Approaches based on quantum transport ${ }^{180}$ have also been proposed, being closer related to the second class of experiments, ${ }^{7}$ which probe the electrical response of DNA self-assembled monolayers in a two terminal setup. Ref. 18 addressed for the first 
time in the context of a quantum transport model the possibility that an electrostatic field with helical symmetry could induce a spin-orbit interaction. An effective onedimensional (1D) Hamiltonian was formulated, assuming that only the $z$-component (along the helical axis) of the electron momentum was not vanishing. Although strong spin-dependent effects were found, it turns out that the model needs to break timereversal symmetry to reveal the spin polarization. This is unsatisfactory from a formal point of view and builds the main motivation to explore extensions of this model not requiring to artificially break any symmetry of the problem. In Ref. 19, the motion of a charge carrier along a helical path including spin-orbit interactions was treated within a tight-binding model. A rather large positive spin polarization was found; however, also here it was necessary to introduce a symmetry breaking interaction through Büttiker probes - in order to achieve spin polarization. The authors also stated that the double-strand structure of the DNA molecule was required to get a non-zero polarization, a result that would impose strong constraints on the molecular systems, where this type of chirality induced spin-dependent effects could be detected.

Apart from these two main lines, in Ref. 21 the emergence of bound states in the electronic system induced by curvature effects was studied, and its possible relation to the spin selectivity analyzed. It is also worth mentioning an earlier work exploring electron transmission/transfer in chiral molecules based on the concept of current transfer. ${ }^{22}$ Furthermore, not directly related to the physical problems at stake in our work but nonetheless relevant for some of the analytical studies proposed here, are investigations of the electronic states in nonplanar geometries, namely 1D curved wires, $2, \sqrt{23}$ helical nanotubes,, 24 bent nanostructures, 2 and the Schrödinger equation in general curvilinear coordinates. ${ }^{[26}$ Besides the simplest case of $1 \mathrm{D}$ curved wires, most of the investigations only address the kinetic-energy part of the Hamiltonian, but do not include spin-orbit interactions in systems with curvature.

In the present study, we generalize our previous work ${ }^{18}$ as well as the work of Guo and Sun $\frac{19}{19}$ in some important aspects. We consider two concentrical helices, as shown in Figure 1 . 
One of the helices, the external one with radius $R_{0}$ and pitch $b$, will provide the helical potential distribution $U(\mathbf{r})$ leading to a helical field $\mathbf{E}(\mathbf{r})$ inducing a SOC. The internal helix, with radius $R<R_{0}$ but the same pitch $b$, describes a possible geometrical path of a charge propagating in the helical field. Also a helical path may be look at the first sight as artificial, it represents a compromise between a purely 1D straight motion and a fully 3D propagation, since it involves a non-trivial geometry (curvature and torsion) but at the same times allows to map the spin transport problem onto a one-dimensional multi-channel tightbinding model. For the sake of simplicity we will consider a distribution of effective point charges along the external helix as done previously in Ref. 18, although our formulation is independent of the specific source of the helical field. This field is felt as an effective, momentum dependent, magnetic field in the rest frame of the charge carrier and gives rise to SOC mirroring the helical symmetry of the system. The effective Schrödinger equation for a spinor wave function of a charge $q$ and spin $\sigma$ moving along this pathway is obtained by an appropriate confinement of the 3D motion along a tubular helix to a 1D motion along the path with curvature $\rho$ and torsion $\tau$.

In addition, we will include two energy levels per site in the tight-binding version of the continuum model, corresponding to the edge orbitals of a molecular monomer building up the helical system. We stress that the two levels do not need to lie on different helices, so that the model only considers transport along a single helical path but with more than one level per site in the tight-binding description. The model can thus be applied to single-helix systems and easily extended to double-helix structures. Our results suggest that two elements are key ingredients to obtain net spin polarization in this class of models: first, including more than one energy level per site (more than one transport pathway) and second, introducing asymmetries in the effective electronic-coupling elements between the different channels.

In the next section, we introduce the general Hamiltonian including SOC in three dimensional (3D) space and provide a derivation of an effective 1D version for the motion along a helical pathway. This has the advantage that we can, firstly, easily include the geometric effects 


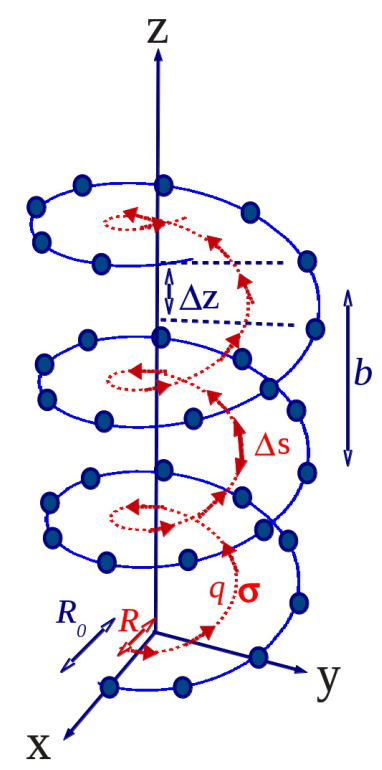

Figure 1: Schematic representation of the system. Along the external helix with radius $R_{0}$ point charges are arranged and build the source of the electrostatic field felt by a charge moving along the internal helical path of radius $R$. The internal helical path is parametrized with the arc length $s$.

arising from the curvature of the helical path and, secondly, take into account the full helical field generated by the external helix.

\section{Local frames and spin-orbit coupling}

In order to set up the appropriate tight-binding model describing the 1D motion of a charge along a helical path, we will first start with a continuum model in 3D space and, after introducing an effective confinement potential, the limit of the motion along a $1 \mathrm{D}$ submanifold will be considered. ${ }^{27}$ The motion of a particle along a helical path in 3D can be described by the following position vector $\mathbf{R}\left(s, q_{1}, q_{2}\right)=\mathbf{x}(s)+q_{1} \mathbf{n}(s)+q_{2} \mathbf{b}(s)$. Here, $\mathbf{x}(s)=(R \cos \phi, R \sin \phi, p \phi)$ with $\phi=s / \sqrt{R^{2}+p^{2}} \equiv s / \kappa$, parametrizes a helix of radius $R$ and pitch $b=2 \pi p$ in $3 \mathrm{D}$, while the coordinates $q_{1}$ and $q_{2}$ are related to a local frame moving along the helix (one can think of a helical tube with e.g. a circular cross section). The normal vector $\mathbf{n}$ and the binormal vector $\mathbf{b}$ are unit vectors orthogonal to each other and 
to the tangent vector $\mathbf{T}(s)=d \mathbf{x}(s) / d s$. These three vectors build a local orthogonal basis, which is closely related to the Frenet-Serret frame $\{\mathbf{T}, \mathbf{N}, \mathbf{B}\}$ (where $\mathbf{N}$ lies in the $x-y$ plane) through the following local rotation mediated by a matrix $\mathbf{U}(\theta)$

$$
\left[\begin{array}{c}
\mathbf{T} \\
\mathbf{n} \\
\mathbf{b}
\end{array}\right]=\left[\begin{array}{ccc}
1 & 0 & 0 \\
0 & \cos \theta & -\sin \theta \\
0 & \sin \theta & \cos \theta
\end{array}\right] \times\left[\begin{array}{c}
\mathbf{T} \\
\mathbf{N} \\
\mathbf{B}
\end{array}\right]
$$

The angle $\theta(s)=\int_{s_{0}}^{s} d u \tau(u)$ reduces to $\theta(s)=\tau s$ in case of a constant external torsion $\tau$. For a helix, the torsion $\tau=p / \kappa^{2}$ and its curvature is $\rho=R / \kappa^{2}$. The introduction of the above rotation mediated by the angle $\theta$ is related to the following fact: If the position of a point on the helical tube is expressed only in terms of the Frenet-Serret frame, it turns out that the basis $\mathbf{e}_{s}=\partial \mathbf{R} / \partial s, \mathbf{e}_{1}=\partial \mathbf{R} / \partial q_{1}, \mathbf{e}_{2}=\partial \mathbf{R} / \partial q_{2}$ is non-orthogonal for $q_{1}^{2}+q_{2}^{2}>0$. This makes the calculation of the kinetic-energy operator and of the SOC operator quite difficult, since the metric tensor is non-diagonal and hence mixed derivatives can appear in the different expressions. The local rotation by the angle $\theta$ overcomes this problem by adapting the local frame at each point along the path $s$ in such a way that the Darboux vector does not have any component on the tangential direction. ${ }^{24}$ Notice that hereby only the normal and binormal vectors are affected by the rotation, while the local tangent vector is the same in both frames. In Cartesian coordinates, the Frenet-Serret basis has the following representation $($ with $\phi=s / \kappa)$ :

$$
\left[\begin{array}{c}
\mathbf{T} \\
\mathbf{N} \\
\mathbf{B}
\end{array}\right]=\left[\begin{array}{ccc}
-\kappa \rho \sin \phi & \kappa \rho \cos \phi & \kappa \tau \\
-\cos \phi & -\sin \phi & 0 \\
\kappa \tau \sin \phi & -\kappa \tau \cos \phi & \kappa \rho
\end{array}\right] \times\left[\begin{array}{c}
\mathbf{e}_{x} \\
\mathbf{e}_{y} \\
\mathbf{e}_{z}
\end{array}\right]=\mathbf{B}(s) \times\left[\begin{array}{c}
\mathbf{e}_{x} \\
\mathbf{e}_{y} \\
\mathbf{e}_{z}
\end{array}\right]
$$

Using the above expressions and after a lengthy but straightforward calculation, the transformation matrix $\mathbf{J}\left(s, q_{1}, q_{2}\right)$ relating the Cartesian unit vectors to the local frame basis 
$\left(\mathbf{e}_{s}, \mathbf{e}_{1}, \mathbf{e}_{2}\right)$ can be found as

$$
\begin{aligned}
\mathbf{J}\left(s, q_{1}, q_{2}\right) & =\mathbf{B}^{-1}(s) \mathbf{U}^{-1}(\theta) \mathbf{A}^{-1}\left(q_{1}, q_{2}\right) \\
& =\left[\begin{array}{ccc}
-\frac{\kappa \rho}{\eta} \sin \phi & -\left(A_{+} \cos k_{-}+A_{-} \cos k_{+}\right) & -\left(A_{-} \sin k_{+}+A_{+} \sin k_{-}\right) \\
\frac{\kappa \rho}{\eta} \cos \phi & -\left(A_{-} \sin k_{+}-A_{+} \sin k_{-}\right) & -\left(A_{+} \cos k_{-}-A_{-} \cos k_{+}\right) \\
\frac{\kappa \tau}{\eta} & -\kappa \rho \sin \theta & \kappa \rho \cos \theta
\end{array}\right] .
\end{aligned}
$$

In this expression, $\eta=1-\rho\left(q_{1} \cos \theta+q_{2} \sin \theta\right)=\sqrt{g}$, with $g$ being the determinant of the metric tensor with elements $G_{l j}=\eta^{2} \delta_{l 1} \delta_{j 1}+\delta_{l 2} \delta_{j 2}+\delta_{l 3} \delta_{j 3}$ in the local basis. $\mathbf{A}\left(q_{1}, q_{2}\right)$ is a diagonal matrix with elements $A_{j j}=\sqrt{G_{j j}}$. Further, $A_{ \pm}=(1 \pm \kappa \tau) / 2$, and $k_{ \pm}=\theta \pm \phi=$ $(\tau \pm(1 / \kappa)) s= \pm(2 s / \kappa) A_{ \pm}$.

\section{Confining to one dimension}

The above equations describe the motion along a helical tube with a cross section whose precise shape can be specified through the local coordinates $q_{1}$ and $q_{2}$. Thus, e.g. a polar representation $q_{1}=r \cos \gamma$ and $q_{2}=r \sin \gamma$ would correspond to a helical tube with a circular cross-section. We are however not interested in this aspect, since our aim is to reduce the 3D motion along the helical tube to a 1D motion along a helical path. Physically this can be realized through the introduction of a transversal confining potential $V_{\lambda}\left(q_{1}, q_{2}\right)$ with strength $\lambda \rightarrow \infty$. In this limit, the precise shape of this potential does not matter, although for the sake of simplicity one can assume it to be a harmonic confinement.

Formally, the Hamilton operator of a particle with spin 1/2 and including SOC will read as

$$
H=K\left(s, q_{1}, q_{2}\right)+V_{\text {pot }}\left(s, q_{1}, q_{2}\right)+\frac{\alpha_{\mathrm{SOC}}}{2}[\sigma \cdot(\mathbf{p} \times \mathbf{E})-\mathbf{E} \cdot(\mathbf{p} \times \sigma)] .
$$

In this equation, $K\left(s, q_{1}, q_{2}\right)$ is the kinetic energy operator and $V_{\text {pot }}\left(s, q_{1}, q_{2}\right)$ includes the previously mentioned transversal confinement potential $V_{\lambda}$ as well as the potential related to the electric field $\mathbf{E}$ in the SOC term, $\alpha_{\mathrm{SOC}}=e \hbar /(2 m c)^{2}$. The vector $\sigma=\left(\sigma_{x}, \sigma_{y}, \sigma_{z}\right)$ contains 
the Pauli matrices. Notice that we have already symmetrized the SOC Hamiltonian in order to have a Hermitian expression in the continuum case. Special care must be taken of the action of the momentum operator on the electric field and the Pauli matrices since in the new local coordinate system the latter will also become dependent on $s, q_{1}$ and $q_{2}$.

The kinetic-energy term $K\left(s, q_{1}, q_{2}\right)$ has been already discussed in the literature (see e.g. Ref. 27), and it can be written using Einstein's sum convention as

$$
\begin{aligned}
K\left(s, q_{1}, q_{2}\right) & =-\frac{\hbar^{2}}{2 m} \frac{1}{\sqrt{g}} \frac{\partial}{\partial_{j}}\left[g^{j} \sqrt{g} \frac{\partial}{\partial_{j}}\right] \\
& =-\frac{\hbar^{2}}{2 m}\left[\frac{1}{\eta} \frac{\partial}{\partial s}\left(\frac{1}{\eta} \frac{\partial}{\partial s}\right)+\sum_{j=1,2}\left(\frac{\partial^{2}}{\partial q_{j}^{2}}+\frac{\partial}{\partial q_{j}} \ln (\eta) \frac{\partial}{\partial q_{j}}\right)\right] .
\end{aligned}
$$

Hereafter $g^{j}$ stands for the contravariant diagonal components of the metric tensor and index $j$ runs over the three local coordinates $s, q_{1}$ and $q_{2}$. In order to preserve the normalization of the wave function when going from the $3 \mathrm{D}$ situation to $1 \mathrm{D}$, the Ansatz $\Psi\left(s, q_{1}, q_{2}\right)=$ $\eta^{-1 / 2} \chi\left(s, q_{1}, q_{2}\right)$ is now performed. This leads to the result

$$
K\left(s, q_{1}, q_{2}\right) \Psi=-\frac{\hbar^{2}}{2 m}\left[\frac{1}{\eta^{3 / 2}} \frac{\partial}{\partial s}\left(\frac{1}{\eta} \frac{\partial}{\partial s}\right) \chi\right]-\frac{\hbar^{2} \rho^{2}}{8 m \eta^{5 / 2}} \chi-\frac{\hbar^{2}}{2 m \eta^{1 / 2}} \sum_{j=1,2}\left(\frac{\partial^{2}}{\partial q_{j}^{2}}\right) \chi
$$

Notice that the second term in the previous expression is a potential energy term with a purely geometric character since it only depends on the curvature of the helix $\rho$.

In a next step we need to consider the SOC term. First, we can write this term as

$$
\begin{aligned}
& H_{\mathrm{SOC}} \Psi=-\mathrm{i} \hbar \alpha_{\mathrm{SOC}}\left\{\sigma \cdot(\nabla \Psi \times \mathbf{E})+\frac{1}{2}[\nabla \cdot(\mathbf{E} \times \sigma)] \Psi\right\} \\
& =\frac{-\mathrm{i}}{\sqrt{\eta}} \hbar \alpha_{\mathrm{SOC}}\left\{\sigma \cdot(\nabla \chi \times \mathbf{E})+\sqrt{\eta} \chi \sigma \cdot\left(\nabla \frac{1}{\sqrt{\eta}} \times \mathbf{E}\right)+\frac{\chi}{2}[\nabla \cdot(\mathbf{E} \times \sigma)]\right\} \\
& =\frac{-\mathrm{i}}{\sqrt{\eta}} \hbar \alpha_{\mathrm{SOC}}\left\{\sigma \cdot(\nabla \chi \times \mathbf{E})+\frac{1}{2}[\nabla \cdot(\mathbf{E} \times \sigma)] \chi\right\} .
\end{aligned}
$$

Note that the second nabla operator does not act beyond the square brackets. Writing explicitly the terms containing the momentum operator acting along the arc $s$ and collecting 
into $H_{\mathrm{SOC}}^{\perp}$ the contributions including $p_{1}$ and $p_{2}$, we can write the SOC Hamiltonian in the following way

$$
H_{\mathrm{SOC}}=\frac{\alpha_{\mathrm{SOC}}}{\sqrt{\eta}}\left[\sigma_{2} E_{1}-\sigma_{1} E_{2}\right] p_{s}-\frac{\mathrm{i}}{2 \sqrt{\eta}} \hbar \alpha_{\mathrm{SOC}} \partial_{s}\left[\sigma_{2} E_{1}-\sigma_{1} E_{2}\right]+\frac{1}{\sqrt{\eta}} H_{\mathrm{SOC}}^{\perp}
$$

Here $\left(E_{s}, E_{1}, E_{2}\right)$ are the components of the electric field written in the local coordinate frame. Without SOC and in the limit $\lambda \rightarrow \infty$ the Hamiltonian consists only of kinetic and potential energy terms and would be easily separable. Thus the wave function $\chi$ would be written as a product $\chi\left(s, q_{1}, q_{2}\right)=\Phi(s) \xi\left(q_{1}, q_{2}\right)$. In the present case this does not seem so obvious since $H_{\mathrm{SOC}}$ still depends on all coordinates $s, q_{1}$ and $q_{2}$. However, in a first approximation, we may consider that in the limit $\lambda \rightarrow \infty$ the kinetic-energy contributions to the transversal motion become much larger than the energy scale associated with the SOC, so that we may use a sort of adiabatic approximation based on the Ansatz $\Phi(s) \xi^{s}\left(q_{1}, q_{2}\right)$, where $\xi^{s}\left(q_{1}, q_{2}\right)$ is a solution of the transversal Schrödinger equation for a given $s$. In this limit, we can perform an approximate separation of variables and set $q_{1}=q_{2}=0$ everywhere in $K\left(s, q_{1}, q_{2}\right)$ and in the other terms related to the longitudinal motion along the helical path. As a result, we arrive at an effective Hamiltonian for the motion along the 1D helical path in the $2 \times 2$ spin space as

$$
\begin{aligned}
H_{1 D} & =\left[-\frac{\hbar^{2}}{2 m} \frac{\partial^{2}}{\partial s^{2}}-\frac{\hbar^{2} \rho^{2}}{8 m}+V(s)\right] \mathbf{1}_{2 \times 2}-\mathrm{i} \hbar \alpha_{\text {SOC }}\left[\sigma_{2} E_{1}-\sigma_{1} E_{2}\right] \frac{\partial}{\partial s} \\
& -\frac{\mathrm{i}}{2} \hbar \alpha_{\mathrm{SOC}} \frac{\partial}{\partial s}\left[\sigma_{2} E_{1}-\sigma_{1} E_{2}\right]
\end{aligned}
$$

where $\mathbf{1}_{2 \times 2}$ is the $2 \times 2$ unit matrix. The second term in the diagonal part of Eq. (9) is, as mentioned previously, a local potential of purely geometric origin related to curvature effects on the helical path. The term $V(s)$ is the electrostatic potential from where the SOC field $\mathbf{E}(s)$ arises. The fourth term is the spin-orbit term and the last term takes into account that the field components as well as the Pauli matrices depend on the arc length. The matrices 
$\sigma_{1}$ and $\sigma_{2}$ are explicitly given by:

$$
\begin{aligned}
\sigma_{1}(s) & =-\left[\begin{array}{cc}
\kappa \rho \sin \theta & A_{+} e^{\mathrm{i} k_{-}}+A_{-} e^{-\mathrm{i} k_{+}} \\
A_{+} e^{-\mathrm{i} k_{-}}+A_{-} e^{\mathrm{i} k_{+}} & -\kappa \rho \sin \theta
\end{array}\right], \\
\sigma_{2}(s) & =\left[\begin{array}{cc}
\kappa \rho \cos \theta & \mathrm{i}\left(A_{+} e^{\mathrm{i} k_{-}}-A_{-} e^{-\mathrm{i} k_{+}}\right) \\
-\mathrm{i}\left(A_{+} e^{-\mathrm{i} k_{-}}-A_{-} e^{\mathrm{i} k_{+}}\right) & -\kappa \rho \cos \theta
\end{array}\right] .
\end{aligned}
$$

The local field components $E_{1}$ and $E_{2}$ (and $E_{s}$, which does not appear in the final expressions) can be expressed in terms of their components in a cylindrical coordinate system

$$
\begin{aligned}
& E_{1}=A_{+}\left(E_{r} \cos \theta+E_{\phi} \sin \theta\right)+A_{-}\left(E_{r} \cos \theta-E_{\phi} \sin \theta\right)-\kappa \rho E_{z} \sin \theta, \\
& E_{2}=-A_{+}\left(E_{\phi} \cos \theta-E_{r} \sin \theta\right)+A_{-}\left(E_{\phi} \cos \theta+E_{r} \sin \theta\right)+\kappa \rho E_{z} \cos \theta, \\
& E_{s}=-\kappa \rho E_{\phi}+\kappa \tau E_{z} .
\end{aligned}
$$

In terms of the cylindrical field components, the expression $\sigma_{2} E_{1}-\sigma_{1} E_{2}$ from Eq. (9) reads

$$
\sigma_{2}(s) E_{1}-\sigma_{1}(s) E_{2}=\left[\begin{array}{cc}
Z & Z_{0} \\
Z_{0}^{\dagger} & -Z
\end{array}\right] \equiv \mathbf{C}(s)
$$

where $Z=\kappa \rho E_{r}$ and $Z_{0}=\kappa e^{-\mathrm{i} \phi}\left[\rho E_{z}+\tau\left(\mathrm{i} E_{r}-E_{\phi}\right)\right]$.

For a helical charge distribution, as that used in the remaining of this paper, the cylindrical components are given by

$$
\begin{aligned}
E_{r}(\phi, z) & =E_{c} \sum_{n, m}\left[\epsilon_{\mathrm{R}}-\cos (\Delta \phi)\right] g_{n, m}^{3}(\phi, z) \\
E_{\phi}(\phi, z) & =E_{c} \sum_{n, m} \sin (\Delta \phi) g_{n, m}^{3}(\phi, z) \\
E_{z}(\phi, z), & =E_{c} \sum_{n, m} \frac{z-n b-m \Delta z}{R_{0}} g_{n, m}^{3}(\phi, z) \\
g_{n, m}(\phi, z) & =\left[1+\epsilon_{\mathrm{R}}^{2}-2 \epsilon_{\mathrm{R}} \cos (\Delta \phi)+\left(\frac{z-n b-m \Delta z}{R_{0}}\right)^{2}\right]^{-\frac{1}{2}},
\end{aligned}
$$


where $\Delta \phi=\phi-(2 \pi / b) m \Delta z$ and $\epsilon_{R}=R / R_{0}$. In the previous equations, the variables $R, \phi$ and $z$ are the corresponding coordinates of a point on the internal helix and the indices $n$ and $m$ run over the discretized external helix. In general, the prefactor $E_{c}$ will depend on the specific physical origin of the local effective charge at a given site and its knowledge requires a detailed microscopic calculation of field strengths and SOC parameters for specific molecular systems. In Ref. 18, we have provided a rough estimate of the order of magnitude of the combined parameter $\hbar \alpha_{\mathrm{SOC}} E_{c} \sim 2-6 \mathrm{meV} n \mathrm{~nm}$. We will adopt similar orders of magnitude in the current study.

\section{Limiting cases}

In the limit of zero torsion $\tau=0$ (planar geometry) and assuming only the $z$-component of the field to be non-vanishing and constant, one gets

$H_{\text {planar }}=\left[-\frac{\hbar^{2}}{2 m} \frac{\partial^{2}}{\partial s^{2}}-\frac{\hbar^{2}}{8 m} \rho^{2}+V(s)\right] \mathbf{1}_{2 \times 2}+\hbar \alpha_{\mathrm{SOC}} E_{z}\left[\begin{array}{cc}0 & e^{-\mathrm{i} \phi}\left(-\mathrm{i} \partial_{s}-\frac{\rho}{2}\right) \\ e^{\mathrm{i} \phi}\left(-\mathrm{i} \partial_{s}+\frac{\rho}{2}\right) & 0\end{array}\right]$,

which is the standard expression for the planar Rashba Hamiltonian on a 1D curved wire.23 In the case that only the radial field component is assumed to be nonzero and constant, we recover the continuous version of the Hamiltonian of Ref. 19 for the single channel case:

$$
\begin{aligned}
H_{\text {radial }} & =\left[-\frac{\hbar^{2}}{2 m} \frac{\partial^{2}}{\partial s^{2}}-\frac{\hbar^{2}}{8 m} \rho^{2}+V(s)\right] \mathbf{1}_{2 \times 2} \\
& +\hbar \alpha_{\text {SOC }} E_{r}\left[\begin{array}{cc}
-\mathrm{i} \kappa \rho \partial_{s} & \mathrm{i} \kappa \tau e^{-\mathrm{i} \phi}\left(-\mathrm{i} \partial_{s}-\frac{1}{2 \kappa}\right) \\
-\mathrm{i} \kappa \tau e^{\mathrm{i} \phi}\left(-\mathrm{i} \partial_{s}+\frac{1}{2 \kappa}\right) & \mathrm{i} \kappa \rho \partial_{s}
\end{array}\right]
\end{aligned}
$$

Notice, however, that in Ref. 19 neither the geometric potential $-\hbar^{2} \rho^{2} / 8 m$ nor the electrostatic potential $V(s)$ have been considered in the calculations. For the typical parameters of the DNA helix, the geometric potential is rather small $(\sim 5-10 \mathrm{meV})$; only for large curvatures its contribution may become relevant. The electrostatic potential $V(s)$, on the 
contrary, must be included for physical consistency, since it is the source of the helical electric field.

\section{Tight-binding Hamiltonian}

Once the Hamiltonian (9) in the continuum approximation has been obtained, the next step is to discretize it and to map it to a tight-binding model parametrized by the arc length. We start with the SOC part expressed in terms of (12)

$$
H_{\mathrm{SOC}}=-\frac{\mathrm{i}}{2} \hbar \alpha_{\mathrm{SOC}}\left[\mathbf{C}(s) \frac{\partial}{\partial s}+\frac{\partial}{\partial s} \mathbf{C}(s)\right] .
$$

Here, the derivatives with respect to the arc length act on everything to their right, including a possible wave function. The discretized derivative reads

$$
\left[\frac{\partial}{\partial s}\right]_{n m}=\frac{1}{2 \Delta s}\left(\delta_{n, m-1}-\delta_{n, m+1}\right) .
$$

Here $\Delta s=\kappa \Delta \phi=\kappa \pi / 5$ is the discretization step along the arc and $\phi=\pi / 5$ is the angular distance between monomers building the helix (for the sake of reference we take the typical value of $\pi / 5$ for a DNA molecule, although other values are obviously possible). Acting with $H_{\mathrm{SOC}}$ onto a spinor wave function, one obtains

$$
\left(H_{\mathrm{SOC}}\right)_{n, n^{\prime}} \Psi_{n^{\prime}}=\frac{\hbar \alpha_{\mathrm{SOC}}}{4 \Delta s}\left[-\mathrm{i}\left(\mathbf{C}_{n}-\mathbf{C}_{n+1}\right) \Psi_{n+1}+\mathrm{i}\left(\mathbf{C}_{n}+\mathbf{C}_{n-1}\right) \Psi_{n-1}\right], \quad \Psi_{n}=\left(\begin{array}{c}
\Psi_{n, \uparrow} \\
\Psi_{n, \downarrow}
\end{array}\right)
$$


With this, the discretized Schrödinger equation resulting from (9) can be written as follows:

$$
\begin{aligned}
E \Psi_{n} & =\epsilon_{n} \Psi_{n}+\mathbf{M}_{n, n+1} \Psi_{n+1}+\mathbf{M}_{n, n-1} \Psi_{n-1}=0 \\
\epsilon_{n} & =-\frac{\hbar^{2} \rho^{2}}{8 m}+V(n), \\
\mathbf{M}_{n, n+1} & =-\frac{\hbar^{2}}{2 m \Delta s^{2}} \mathbf{1}_{2 \times 2}-\mathrm{i} \frac{\hbar \alpha_{\mathrm{SOC}}}{4 \Delta s}\left(\mathbf{C}_{n}+\mathbf{C}_{n+1}\right) \equiv\left[\begin{array}{ll}
W_{n, n+1} & D_{n, n+1} \\
D_{n, n+1}^{*} & W_{n, n+1}^{*}
\end{array}\right], \\
\mathbf{M}_{n, n-1} & =\left(\mathbf{M}_{n-1, n}\right)^{\dagger} .
\end{aligned}
$$

Notice that the spin-conserving nearest-neighbor electronic coupling $W_{n, n+1}=-\hbar^{2} /\left(2 m \Delta s^{2}\right)-$ $\mathrm{i}\left(\hbar \alpha_{\mathrm{SOC}} / 4 \Delta s\right)\left(Z_{n}+Z_{n+1}\right)$ is renormalized by the diagonal blocks of the SOC Hamiltonian, which will clearly influence the effective band width of the electronic spectrum. The spin-flip hopping term reads $D_{n, n+1}=-\mathrm{i}\left(\hbar \alpha_{\mathrm{SOC}} / 4 \Delta s\right)\left(\left[Z_{0}\right]_{n}+\left[Z_{0}\right]_{n+1}\right)$.

Thanks to the last identity of Eq. (19), the corresponding Hamiltonian is indeed Hermitian

$$
H_{1 D}=\sum_{n, \sigma} \epsilon_{n} c_{n, \sigma}^{\dagger} c_{n, \sigma}+\sum_{n}\left[\left(c_{n, \uparrow}^{\dagger}, c_{n, \downarrow}^{\dagger}\right) \mathbf{M}_{n, n+1}\left(\begin{array}{c}
c_{n+1, \uparrow} \\
c_{n+1, \downarrow}
\end{array}\right)+\left(c_{n, \uparrow}^{\dagger}, c_{n, \downarrow}^{\dagger}\right) \mathbf{M}_{n, n-1}\left(\begin{array}{c}
c_{n-1, \uparrow} \\
c_{n-1, \downarrow}
\end{array}\right)\right] .
$$

This Hamiltonian preserves time reversal symmetry generated by the operator $\mathcal{T}=-i \sigma_{y} \otimes$ $\mathbf{1}_{N \times N}$, where $N$ is the number of sites in the discretized tight-binding model and $\mathbf{1}_{N \times N}$ is the unit matrix in the Hilbert space generated by the $N$ localized orbitals.

The present model can then be easily extended to include two orbitals - calling them HOMO (H) and LUMO (L) - per lattice site. If we neglect SOC between orbitals of different type and, moreover, assume that the effective SOC parameter $\alpha$ may depend on the specific electronic state (although not on the lattice site $n$ ), then the generalization of the previous 
Hamiltonian is straightforward

$$
\begin{aligned}
H_{1 D} & =\sum_{k=H, L} \sum_{n, \sigma} \epsilon_{n}^{k} c_{n, k, \sigma}^{\dagger} c_{n, k, \sigma}+V_{\mathrm{HL}} \sum_{n, \sigma}\left[c_{n, H, \sigma}^{\dagger} c_{n, L, \sigma}+c_{n, L, \sigma}^{\dagger} c_{n, H, \sigma}\right] \\
& +\sum_{k=H, L} \sum_{n}\left[\left(c_{n, k, \uparrow}^{\dagger}, c_{n, k, \downarrow}^{\dagger}\right) \mathbf{M}_{n, n+1}^{k}\left(\begin{array}{c}
c_{n+1, k, \uparrow} \\
c_{n+1, k, \downarrow}
\end{array}\right)+\left(c_{n, k, \uparrow}^{\dagger}, c_{n, k, \downarrow}^{\dagger}\right) \mathbf{M}_{n, n-1}^{k}\left(\begin{array}{c}
c_{n-1, k, \uparrow} \\
c_{n-1, k, \downarrow}
\end{array}\right)\right]
\end{aligned}
$$

In this equation, $V_{\mathrm{HL}}$ is the local coupling between the two levels $H$ and $L$ at site $n$. Notice that the nearest-neighbor electronic couplings $W_{n, n \pm 1}^{k}$ (included in $\mathbf{M}_{n, n \pm 1}^{k}$, see Eq. 19 depend now on the specific orbital: $W_{n, n \pm 1}^{H, L}=V_{\mathrm{H}, \mathrm{L}}-\mathrm{i}\left(\alpha_{\mathrm{H}, \mathrm{L}} / E_{c}\right)\left(Z_{n}+Z_{n \pm 1}\right)$, where $\alpha_{\mathrm{H}, \mathrm{L}}$ is now considered as an effective parameter with the dimensions energy $\times$ length.

We have also introduced a staggered contribution to the site energies $\epsilon_{n}^{k}=-\hbar^{2} \rho^{2} / 8 m+V(n)+$ $\Delta_{k}(-1)^{n}$ which opens a band gap. A gap opening can also be achieved with a large $V_{\mathrm{HL}}$; however, in order to keep the orders of magnitude of the electronic coupling parameters in a realistic range of few tens of $\mathrm{meV}$, we will keep the $\Delta_{k}(-1)^{n}$ contribution with $\Delta_{k}=30 \mathrm{meV}$.

\section{Transport properties}

Spin-dependent transport in the tight-binding model in Eq. (21) can be represented as a charge transport model in a 4-legs ladder, where each leg corresponds to a molecular orbital ( $H$ or $L)$ and to a given spinor component $(\uparrow$ and $\downarrow)$. It is clear that the specific transport mechanism will in general depend on the molecular system and also be influenced by the environmental conditions, e.g. single molecule vs. molecule embedded in a self-assembled monolayer or dry vs. solvent conditions. Being aware of its potential limitations, we will consider transport in the context of the Landauer approach, which provides a simple framework to analyze the influence of different parameters on the spin polarization. Decoherence to mimic hopping transport can be introduced via Büttiker probes ${ }^{28}$ or by directly formulating the problem in terms of master equations. However, we are interested in exploring the possibility of inducing a spin polarization without the need of decoherence as a key ele- 
ment, so that we limit ourselves to compute the zero-bias transmission function $T(E)$ (linear conductance) for our model.

The Hamiltonian (21) needs to be extended to include the coupling to the electrodes for which the Hamiltonian reads $H_{\text {leads. }}$ Along similar lines as in Ref. 18, we consider four independent $\mathcal{L}$ (left)- and four independent $\mathcal{R}$ (right)-leads, each of them standing for a spin channel connected to a specific molecular orbital $(H, L)$ and being represented by a semiinfinite tight-binding chain.

The transmission function is given by the expression: $T(E)=\operatorname{Tr}\left\{\mathbf{G}^{a}(E) \Gamma^{\mathrm{R}} \mathbf{G}^{r}(E) \Gamma^{\mathrm{L}}\right\}$. Here $\mathbf{G}^{r(a)}(E)$ are retarded (advanced) matrix Green's functions for the scattering region including the influence of $H_{\text {leads }}$ via retarded self-energy matrices $\Sigma^{r, \mathcal{L}}(E)$ and $\Sigma^{r, \mathcal{R}}(E)$. The retarded Green's function matrix can be determined via Dyson's equation $\left(\mathbf{G}^{r}\right)^{-1}(E)=(E+\mathrm{i} \eta) \mathbf{1}-$ $\mathbf{H}-\Sigma^{r, \mathcal{L}}(E)-\Sigma^{r, \mathcal{R}}(E)$. The spectral functions $\Gamma^{\mathcal{L}, \mathcal{R}}$ of the left and right electrodes are related to the self-energies via $\Gamma^{\mathcal{L}, \mathcal{R}}=-2 \operatorname{Im} \Sigma^{r, \mathcal{L}, \mathcal{R}}(E)$. The only eight non-vanishing elements of the spectral functions are $\Gamma_{\uparrow, L, H}^{\mathcal{L}}, \Gamma_{\downarrow, L, H}^{\mathcal{L}}, \Gamma_{\uparrow, L, H}^{\mathcal{R}}, \Gamma_{\downarrow, L, H}^{\mathcal{R}}$. These quantities are in general energydependent and can be computed analytically for semi-infinite chains (see, e.g., Ref. 29). Using the previous expressions and approximations, the total transmission function for the system can be written as

$$
T(E)=T_{\uparrow \uparrow}(E)+T_{\downarrow \uparrow}(E)+T_{\uparrow \downarrow}(E)+T_{\downarrow \downarrow}(E)
$$

where

$$
\begin{aligned}
& T_{\uparrow \uparrow}(E)=\Gamma_{\uparrow, L}^{\mathcal{L}}\left[\Gamma_{\uparrow, L}^{\mathcal{R}}\left|G_{1 \uparrow, N \uparrow}\right|^{2}+\Gamma_{\uparrow, H}^{\mathcal{R}}\left|G_{1 \uparrow, 2 N \uparrow}\right|^{2}\right]+\Gamma_{\uparrow, H}^{\mathcal{L}}\left[\Gamma_{\uparrow, L}^{\mathcal{R}}\left|G_{N+1 \uparrow, N \uparrow}\right|^{2}+\Gamma_{\uparrow, H}^{\mathcal{R}}\left|G_{N+1 \uparrow, 2 N \uparrow}\right|^{2}\right], \\
& T_{\uparrow \downarrow}(E)=\Gamma_{\uparrow, L}^{\mathcal{L}}\left[\Gamma_{\downarrow, L}^{\mathcal{R}}\left|G_{1 \uparrow, N \downarrow}\right|^{2}+\Gamma_{\downarrow, H}^{\mathcal{R}}\left|G_{1 \uparrow, 2 N \downarrow}\right|^{2}\right]+\Gamma_{\uparrow, H}^{\mathcal{L}}\left[\Gamma_{\downarrow, H}^{\mathcal{R}}\left|G_{N+1 \uparrow, 2 N \downarrow}\right|^{2}+\Gamma_{\downarrow, L}^{\mathcal{R}}\left|G_{N+1 \uparrow, N \downarrow}\right|^{2}\right], \\
& T_{\downarrow \downarrow}(E)=\Gamma_{\downarrow, L}^{\mathcal{L}}\left[\Gamma_{\downarrow, L}^{\mathcal{R}}\left|G_{1 \downarrow, N \downarrow}\right|^{2}+\Gamma_{\downarrow, H}^{\mathcal{R}}\left|G_{1 \downarrow, 2 N \downarrow}\right|^{2}\right]+\Gamma_{\downarrow, H}^{\mathcal{L}}\left[\Gamma_{\downarrow, L}^{\mathcal{R}}\left|G_{N+1 \downarrow, N \downarrow}\right|^{2}+\Gamma_{\downarrow, H}^{\mathcal{R}}\left|G_{N+1 \downarrow, 2 N \downarrow}\right|^{2}\right], \\
& T_{\downarrow \uparrow}(E)=\Gamma_{\downarrow, L}^{\mathcal{L}}\left[\Gamma_{\uparrow, L}^{\mathcal{R}}\left|G_{1 \downarrow, N \uparrow}\right|^{2}+\Gamma_{\uparrow, H}^{\mathcal{R}}\left|G_{1 \downarrow, 2 N \uparrow}\right|^{2}\right]+\Gamma_{\downarrow, H}^{\mathcal{L}}\left[\Gamma_{\uparrow, H}^{\mathcal{R}}\left|G_{N+1 \downarrow, 2 N \uparrow}\right|^{2}+\Gamma_{\uparrow, L}^{\mathcal{R}}\left|G_{N+1 \downarrow, N \uparrow}\right|^{2}\right] .
\end{aligned}
$$


In the previous equations, $G_{n \sigma, m \nu}(E)$ with $\sigma, \nu=\uparrow, \downarrow$ are matrix elements of the previously defined retarded Green's function of the SOC-active region including the influence of the $\mathcal{L}$ and $\mathcal{R}$-electrodes via appropriate self-energies. Each contribution in Eq. 22 can be related to a different transport process with or without spin flip. Thus, all contributions included in $T_{\uparrow \uparrow}$ and $T_{\downarrow \downarrow}$ are related to processes taking place only in the spin-up or spin-down channels, respectively, while $T_{\uparrow \downarrow}$ and $T_{\downarrow \uparrow}$ involve all processes flipping the electron spin. With the help of the previously introduced transmission components, we can define a spin polarization (SP) as:

$$
P(E)=\frac{1}{T(E)}\left[T_{\uparrow \uparrow}(E)+T_{\downarrow \uparrow}(E)-T_{\uparrow \downarrow}(E)-T_{\downarrow \downarrow}(E)\right] .
$$

Finally, an energy-averaged SP can be defined as $\langle P\rangle_{E}=\left[\left\langle T_{\uparrow \uparrow}+T_{\downarrow \uparrow}\right\rangle_{E}-\left\langle T_{\uparrow \downarrow}+T_{\downarrow \downarrow}\right\rangle_{E}\right] /\langle T\rangle_{E}$. When computing it, we use only the hole-like contributions of the $P(E)$ plot, i.e., the $E<0$ sector, so that $\langle\ldots\rangle_{E}=\left(2 V_{0}\right)^{-1} \int_{-2 V_{0}}^{0} d E(\ldots)$. Here, $V_{0}$ is determined by diagonalizing the Hamiltonian of Eq. (21), calculating the band width $\Delta E$ and taking $V_{0}=\Delta E / 4$. This choice guarantees that we include all the relevant molecular electronic states in the calculation of the transmission function. $V_{0}$ is also taken as the effective band width of the electrodes. Thus, for the semi-infinite linear chain electrodes with a single orbital per side, the non-vanishing matrix elements of the spectral functions $\Gamma^{\mathcal{L}, \mathcal{R}}(E)$ are given by the standard Anderson-Newns expressions $\Gamma_{\uparrow, \downarrow, L, H}^{\mathcal{L}, \mathcal{R}}(E)=V_{0} \sqrt{1-\left(E / 2 V_{0}\right)^{2}}$ for $\left|E / 2 V_{0}\right|<1$ and zero otherwise, where the site energies have been set equal to zero.

\section{Results and discussion}

We address in this section two issues: (i) Is it possible to obtain a non-zero spin polarization without including decoherence in the model formulation? (ii) What is the influence of having more than one molecular orbital per site in the tight-binding representation?

Concerning the typical order of magnitude of the spin-orbit interaction as induced by the 


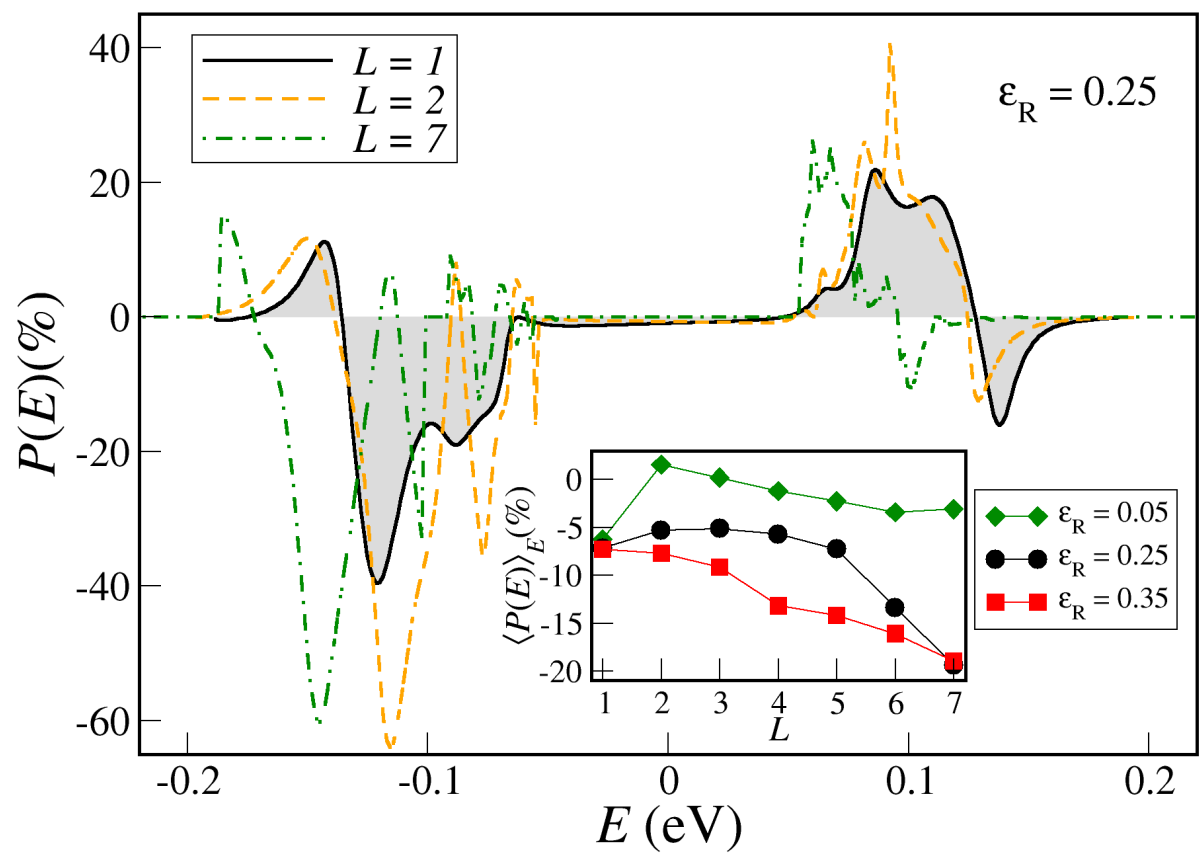

Figure 2: Spin polarization for an unpolarized incoming state and for three different numbers of turns $L=1,2$ and 7 of the helix. The parameter $\epsilon_{R}$ has been set to 0.25 . The SP is mostly negative over the hole band $(E<0)$ and mostly positive over the electronic band $(E>0)$. The inset shows the dependence of the energy-averaged SP on the number of helical turns and several values of $\epsilon_{R}=R / R_{0}$. The absolute value increases with a larger $\epsilon_{\mathrm{R}}$. For larger $L$ we may expect an oscillatory behavior to set in. Other parameters are $\alpha_{\mathrm{H}}=0.2 \mathrm{meV} \mathrm{nm}$, $\alpha_{\mathrm{L}}=2 \mathrm{meV} \mathrm{nm}, V_{\mathrm{L}}=10 \mathrm{meV}, V_{\mathrm{H}}=90 \mathrm{meV}$ and $V_{\mathrm{HL}}=50 \mathrm{meV}$.

helical field, we have already provided a rough estimate of its strength in a previous study; 18 in the present investigation we are going to use similar values, being aware that a more accurate estimation would require a separate first-principle study of the electronic structure of the molecules. Along this paper, if not stated otherwise, we will keep fixed the bare electronic-coupling parameters: $V_{\mathrm{L}}=10 \mathrm{meV}, V_{\mathrm{H}}=90 \mathrm{meV}$ and $V_{\mathrm{HL}}=50 \mathrm{meV}$. These values have typical orders of magnitude as for a DNA molecule. We remark, however, that we are not addressing a specific molecular system, but are deriving a generic model and investigating its main properties.

For the following discussion we will also introduce two parameters, namely $\eta_{\mathrm{SOC}}=\alpha_{\mathrm{H}} / \alpha_{\mathrm{L}}$ and $\eta_{\text {elec }}=V_{\mathrm{H}} / V_{\mathrm{L}}$ measuring, respectively, the relative asymmetries of the SOC and of the nearest-neighbor tight-binding hopping elements for the $H$ and $L$ levels. 
In Figure 2, the spin polarization for an incoming unpolarized state is shown as a function of the injection energy for different lengths and for the case of an array of helically distributed (negative) charges. The first interesting feature of Figure 2 is that a non-zero SP can be obtained with absolute values which can be as large as $60 \%$ for certain energies. These results suggest that decoherence may not be a key element to reveal a spin polarization. 19 Moreover, it seems necessary (although not sufficient, as discussed below) to have more than one transport channel. This can be implemented in the model either as a double-strand structure $^{\sqrt{19}}$ or as a single strand with more than one electronic level per site. Notice also that despite of the oscillations of the SP as a function of the energy, there is a clear tendency to have a negative polarization for the electron bands $(E<0)$ and positive polarization for the hole bands $(E>0)$. This behavior does not depend much on the length of the helical system. The inset of Figure 2 shows the dependence of the energy-averaged SP on the number of helical turns $L$ for three different values of the parameter $\epsilon_{\mathrm{R}}=R / R_{0}$. This parameter quantifies the difference between the radii of the internal and external helices and it is thus a measure for the radius of curvature of the transport path: for $\epsilon_{\mathrm{R}} \ll 1$ the transport pathway approaches a straight line (for a fixed radius of the external helix). For larger $\epsilon_{\mathrm{R}}$, the average $\mathrm{SP}$ is negative for all $L$ and its absolute value progressively increases with $L$. The effect becomes stronger with increasing $\epsilon_{\mathrm{R}}$, since the helical field will be felt stronger the closer the charge transport path is to the external helical charge distribution. The negative average value indicates that the spin-down channel is easier transmitted than the spin-up channel over the averaged energy window. For $\epsilon_{\mathrm{R}}=0.05$, on the contrary, the SP varies in a considerably weaker way with increasing length and its absolute value stays below $5 \%$. This is an indication that the intrinsic geometry of the path, its helical structure, is playing an important role in influencing the spin polarization.

In Figure 3, the quantities $\xi_{\text {up }}=T_{\uparrow \uparrow}-T_{\downarrow \uparrow}$ and $\xi_{\text {down }}=T_{\downarrow \downarrow}-T_{\uparrow \downarrow}$ are plotted, in order to illustrate the relative amount of spin-conserving and spin-flip processes in the outgoing spinup and spin-down channels. Hence, for energy regions where $\xi_{\sigma}$ is positive, spin-conserving 

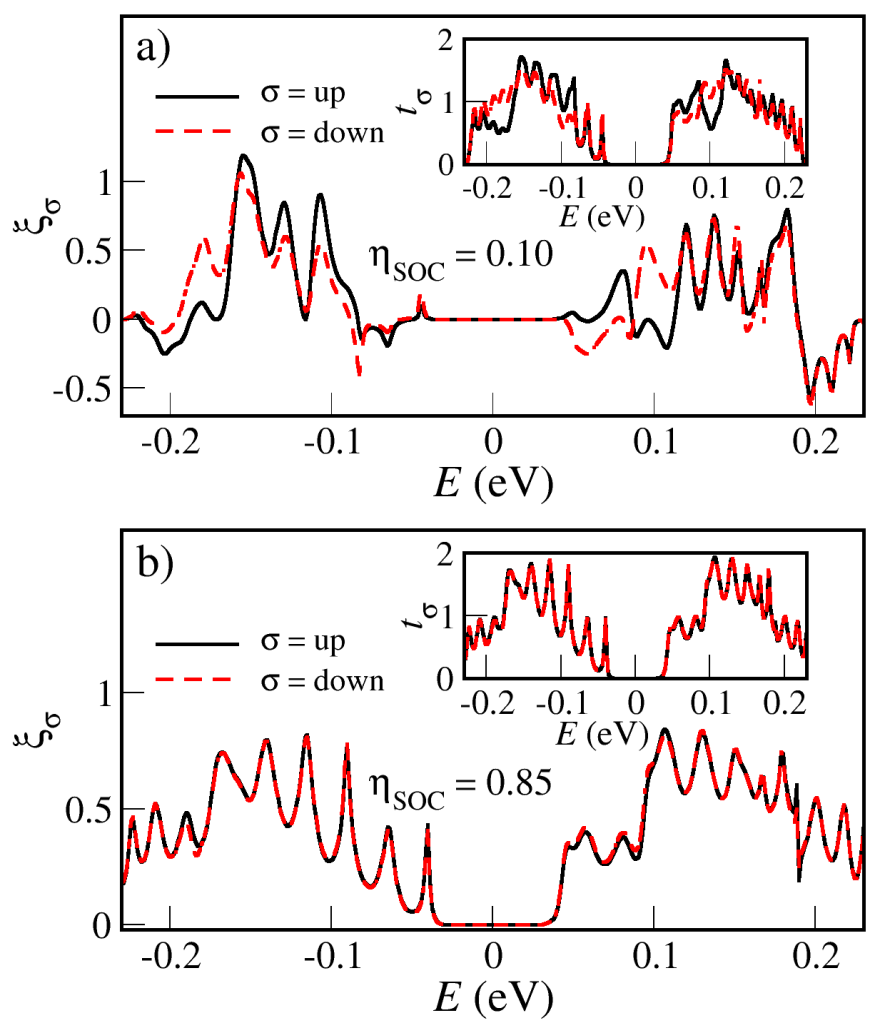

Figure 3: Energy-resolved ratios of the transmission function for the outgoing spin-up channel, $\xi_{\text {up }}=T_{\uparrow \uparrow}-T_{\downarrow \uparrow}$, and spin-down channel, $\xi_{\text {down }}=T_{\downarrow \downarrow}-T_{\uparrow \downarrow}$. Insets show the total transmission $t_{\text {up }}=T_{\uparrow \uparrow}+T_{\downarrow \uparrow}$ and $t_{\text {down }}=T_{\downarrow \downarrow}+T_{\uparrow \downarrow}$, respectively. The calculations have been performed for equal electronic-coupling elements $V_{\mathrm{L}}=V_{\mathrm{H}}=90 \mathrm{meV}$, in order to highlight the influence of asymmetries in the SOC parameters $\eta_{\mathrm{SOC}}=\alpha_{\mathrm{H}} / \alpha_{\mathrm{L}}$, as indicated on each plot. For stronger asymmetry [panel a)] in the SOC coupling, there the differences between $\xi_{\text {up }}$ and $\xi_{\text {down }}$ are stronger, which eventually makes spin filtering and spin aligning possible. The remaining parameters are $\alpha_{\mathrm{L}}=2 \mathrm{meV} \mathrm{nm}, V_{\mathrm{HL}}=50 \mathrm{meV}, \epsilon_{\mathrm{r}}=0.25$, and $L=2$ helical turns.

processes are dominating, while for $\xi_{\sigma}$ negative, spin-flip events dominate. In addition, the insets of Figure 3 show the total transmission of each spin component. Notice that the difference between the curves of $t_{\mathrm{up}}$ and $t_{\text {down }}$ is proportional to the spin polarization (after division by the total transmission $T(E)$, see also Eq. 23). The results presented in Figure 3 are calculated for symmetric electronic coupling $V_{\mathrm{L}}=V_{\mathrm{H}}$ and for two different values of the SOC ratio $\eta_{\mathrm{SOC}} \equiv \alpha_{\mathrm{H}} / \alpha_{\mathrm{L}}=0.1$ and 0.85 , to point out the difference between strong and a weak asymmetry in the SOC parameters of the H- and L-states. For a strong asymmetry $\eta_{\mathrm{SOC}}=0.1$ - leading to net spin polarization over most of the probed energies - a large 
degree of spin-flip is found mainly near the band edges of both the HOMO and LUMO manifolds. For other energies, the spin conserving processes dominate, i.e. $\xi_{\sigma=\text { up,down }}>0$. However, the magnitude of $\xi_{\text {up }}$ and $\xi_{\text {down }}$ is different and thus a non-zero polarization resulting from an interplay between spin-dependent backscattering, a measure of which is the total backscattering given by $R=4-\left(t_{\mathrm{up}}+t_{\text {down }}\right)$, and spin-flip processes can be obtained. On the contrary, for the case $\eta_{\mathrm{SOC}}=0.85$ spin-conserving transmission processes dominate over spin-flips at all energies. Moreover, there is almost no difference between the backscattering of the two spin components nor between $\xi_{\text {up }}$ and $\xi_{\text {down }}$, so that the polarization is almost negligible. In the limiting case $\eta_{\mathrm{SOC}}=1$, the spin polarization identically vanishes.

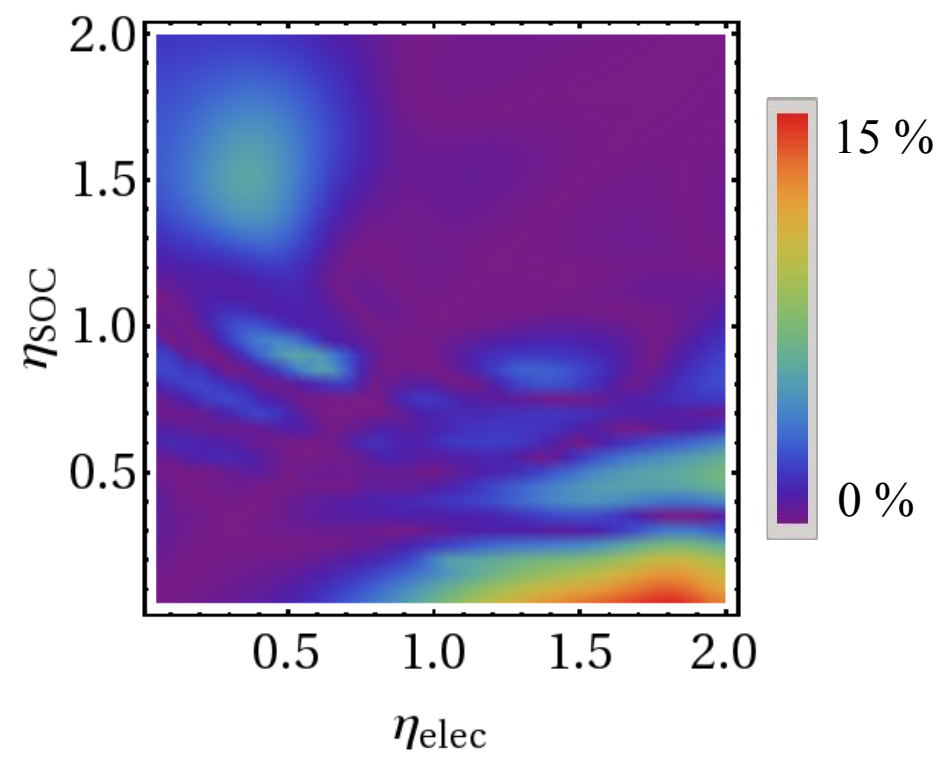

Figure 4: Density plot showing the absolute value of the average spin polarization $\langle P(E)\rangle_{E}$ as a function of the asymmetry ratios $\eta_{\mathrm{SOC}}=\alpha_{\mathrm{H}} / \alpha_{\mathrm{L}}$ and $\eta_{\text {elec }}=V_{\mathrm{H}} / V_{\mathrm{L}}$. Parameters are $\alpha_{\mathrm{L}}=2 \mathrm{meV} \mathrm{nm}, V_{\mathrm{L}}=30 \mathrm{meV}, V_{\mathrm{HL}}=50 \mathrm{meV}, \epsilon_{\mathrm{r}}=0.25$, and $L=2$ helical turns.

To provide a global overview of the effect of the previously mentioned asymmetries in the electronic coupling and spin-orbit interaction, we show in Figure 4 a density plot of the energy-average polarization as a function of the two asymmetry parameters $\eta_{\text {SOC }}$ and $\eta_{\text {elec }}$ - We only plot the absolute value of the average polarization, since our main goal is to 
show the regions where a polarization can be obtained and those where it will either vanish or become too small. From the figure it becomes clear that the spin polarization is very small around the main diagonal where $\eta_{\mathrm{SOC}}=\eta_{\text {elec }}$, i.e., for the fully symmetric case. The largest polarizations are obtained in the lower right sector and in the upper left sector of the diagram, where the asymmetries between both parameters become largest.

We can gain a qualitative understanding of the vanishing polarization along the main diagonal of Figure 4 by the following reasoning. For the continuum model, Eq. (9), a unitary transformation $U(s)=\exp \left[-\mathrm{i}\left(m \alpha_{\mathrm{SOC}} / \hbar\right) \int^{s} d u \mathbf{C}(u)\right]$ can be performed that eliminates the SOC term from the Hamiltonian. $\frac{19}{\sqrt{19}}$ This is the reason why a single-channel model without symmetry breaking does not yield spin polarization. However, if more than one channel are present, e.g. $\mathrm{H}$ and $\mathrm{L}$, then the Hamiltonian would be transformed by the direct sum $U_{\mathrm{H}} \oplus U_{\mathrm{L}}$. This leads to couple terms like $V_{\mathrm{HL}} U_{\mathrm{H}}^{\dagger} U_{\mathrm{L}}$, which are only diagonal in the spin degrees of freedom if $m_{\mathrm{H}} \alpha_{\mathrm{H}} / m_{\mathrm{L}} \alpha_{\mathrm{L}} \sim V_{\mathrm{L}} \alpha_{\mathrm{H}} / V_{\mathrm{H}} \alpha_{\mathrm{L}}=1$, which is the case for $\eta_{\mathrm{SOC}}=\eta_{\text {elec }}$. Only if there is a stronger asymmetry in the ratio $\eta_{\mathrm{SOC}} / \eta_{\mathrm{elec}}$, the SOC may remain effective and a non-trivial behavior may be expected, as observed in the regions off the diagonal in Figure 4

\section{Summary}

We have derived an effective 1D Hamiltonian to describe the propagation of a charge carrier with spin along a helical pathway under the influence of spin-orbit interaction. The SOC is assumed to be induced by a field of point charges arranged along a concentric external helix. It has been shown that a net spin polarization of an incoming unpolarized state can be achieved using realistic parameters for the electronic structure. For up to seven helical turns, a progressive increase of the (negative) polarization with length was found, although the computed values are smaller than those measured. The spin polarization results from an interplay between spin-dependent backscattering and spin-flip processes. This behavior is 
however strongly weakened upon reducing the different between the two orbital-dependent SOC parameters, so that in the limit of fully symmetric coupling to both molecular levels $H$ and $L$, only spin conserving processes take place and, moreover, the spin polarization identically vanishes. Interestingly, our model does not require the presence of decoherence to yield a non-zero spin polarization. However, whether decoherence is an important ingredient or not requires further inquiry. In our case, the non-vanishing polarization emerges when (i) more than one transport channel is included in the model description, and related to it, when (ii) the electronic states display strong asymmetries in both their electronic coupling and the corresponding SOC parameters.

\section{Associated content}

\section{Supporting information}

None

\section{Author Information}

Corresponding Author

Rafael Gutierrez

E-mail: rafael.gutierrez@tu-dresden.de

Institute for Materials Science

Dresden University of Technology

01062 Dresden, Germany

\section{Acknowledgement}

The authors thank Ron Naaman for very enlightening discussions on spin-dependent effects in helical systems. This work was supported by the German Academic Exchange Service 
(DAAD - project reference nr. 54367888) and by Ministerio de Economía y Comptetitividad (MINECO - PRI-AIBDE-2011-0.927) within the joint program Acciones Integradas. Computational resources were provided by the ZIH at TU-Dresden. T.B. thanks the International Max Planck Research School Dynamical Processes in Atoms, Molecules and Solids for financial support. E.D, C.G. and F.D-A were further supported by MINECO (MAT 2010-17180), and research of C.G. was funded by a PICATA postdoctoral fellowship from the Moncloa Campus of International Excellence (UCM-UPM). We gratefully acknowledge support from the German Excellence Initiative via the Cluster of Excellence EXC 1056 "Center for Advancing Electronics Dresden" (cfAED). This research was partially supported by World Class University program funded by the Ministry of Education, Science and Technology through the National Research Foundation of Korea (R31-10100).

\section{Additional notes}

\section{References}

(1) Xiong, Z. H.; Wu, D.; Valy Vardeny, Z.; Shi, J. Giant Magnetoresistance In Organic Spin-valves. Nature 2004, 427, 821-824.

(2) Mooser, S.; Cooper, J. F. K.; Banger, K. K.; Wunderlich, J.; Sirringhaus, H. Spin Injection and Transport in a Solution-processed Organic Semiconductor at Room Temperature. Phys. Rev. B 2012, 85, 235202-1-235202-7.

(3) Grünewald, M.; Wahler, M.; Schumann, F.; Michelfeit, M.; Gould, C.; Schmidt, R.; Würthner, F.; Schmidt, G.; Molenkamp, L. W. Tunneling Anisotropic Magnetoresistance in Organic Spin Valves. Phys. Rev. B 2011, 84, 125208-1-125208-5.

(4) Li, K.-S.; Chang, Y.-M.; Agilan, S.; Hong, J.-Y.; Tai, J.-C.; Chiang, W.-C.; Fuku- 
tani, K.; Dowben, P. A.; Lin, M.-T. Organic Spin Valves with Inelastic Tunneling Characteristics. Phys. Rev. B 2011, 83, 172404-1-172404-4.

(5) Sun, D.; Yin, L.; Sun, C.; Guo, H.; Gai, Z.; Zhang, X.-G.; Ward, T. Z.; Cheng, Z.; Shen, J. Giant Magnetoresistance in Organic Spin Valves. Phys. Rev. Lett. 2010, 104, 236602-1-236602-4.

(6) Göhler, B.; Hamelbeck, V.; Markus, T. Z.; Kettner, M.; Hanne, G. F.; Vager, Z.; Naaman, R.; Zacharias, H. Spin Selectivity in Electron Transmission Through SelfAssembled Monolayers of Double-Stranded DNA. Science 2011, 331, 894-897.

(7) Xie, Z.; Markus, T. Z.; Cohen, S. R.; Vager, Z.; Gutierrez, R.; Naaman, R. Spin Specific Electron Conduction through DNA Oligomers. Nano Lett. 2011, 11, 4652-4655.

(8) Ray, K.; Ananthavel, S. P.; Waldeck, D. H.; Naaman, R. Asymmetric Scattering of Polarized Electrons by Organized Organic Films of Chiral Molecules. Science 1999, 283, 814-816.

(9) Carmeli, I.; Skakalova, V.; Naaman, R.; Vager, Z. Magnetization of Chiral Monolayers of Polypeptide: A Possible Source of Magnetism in Some Biological Membranes. Angew. Chem. Int. Ed. 2002, 41, 761-764.

(10) Carmeli, I.; Leitus, G.; Naaman, R.; Reich, S.; Vager, Z. Magnetism Induced by the Organization of Self-assembled Monolayers. J. Chem. Phys. 2003, 118, 10372-10375.

(11) Vager, Z.; Naaman, R. Surprising Electronic-Magnetic Properties of Close-Packed Organized Organic Layers. Chem. Phys. 2002, 281, 305 - 309.

(12) Naaman, R.; Vager, Z. New Electronic and Magnetic Properties Emerging from Adsorption of Organized Organic Layers. Phys. Chem. Chem. Phys. 2006, 8, 2217-2224. 
(13) Ray, S. G.; Daube, S. S.; Leitus, G.; Vager, Z.; Naaman, R. Chirality-Induced SpinSelective Properties of Self-Assembled Monolayers of DNA on Gold. Phys. Rev. Lett. 2006, 96, 036101-1-036101-4.

(14) Wei, J. J.; Schafmeister, C.; Bird, G.; Paul, A.; Naaman, R.; Waldeck, D. H. Molecular Chirality and Charge Transfer through Self-Assembled Scaffold Monolayers. J. Phys. Chem. B 2005, 110, 1301-1308.

(15) Naaman, R.; Vager, Z. Spin Selective Electron Transmission Through Monolayers of Chiral Molecules. In Electronic and Magnetic Properties of Chiral Molecules and Supramolecular Architectures; Naaman, R., Beratan, D. N., Waldeck, D., Eds.; Topics in Current Chemistry; Springer Berlin / Heidelberg, 2011, 298, Chapter 91, 237-257.

(16) Yeganeh, S.; Ratner, M. A.; Medina, E.; Mújica, V. Chiral Electron Transport: Scattering through Helical Potentials. J. Chem. Phys. 2009, 131, 014707-1-014707-9.

(17) Medina, E.; López, F.; Ratner, M.; Mújica, V. Chiral Molecular Films as Electron Polarizers and Polarization Modulators. European Phys. Lett. 2012, 99, 17006-p1$17006-\mathrm{p} 5$.

(18) Gutierrez, R.; Díaz, E.; Naaman, R.; Cuniberti, G. Spin-selective Transport through Helical Molecular Systems. Phys. Rev. B 2012, 85, 081404(R)-1-081404(R)-4.

(19) Guo, A. M.; Sun, Q. F. Spin-Selective Transport of Electrons in DNA Double Helix. Phys. Rev. Lett. 2012, 108, 218102-1-218102-4.

(20) Guo, A.-M.; Sun, Q. F. Enhanced spin-polarized transport through DNA double helix by gate voltage. Phys. Rev. B 2012, 86, 035424-1-035424-5.

(21) Vager, D.; Vager, Z. Spin Order without Magnetism: a New Phase of Spontaneously Broken Symmetry in Condensed Matter. Phys. Lett. A 2012, 376, 1895-1897. 
(22) Skourtis, S. S.; Beratan, D. N.; Naaman, R.; Nitzan, A.; Waldeck, D. H. Chiral Control of Electron Transmission through Molecules. Phys. Rev. Lett. 2008, 101, 238103-1$238103-4$.

(23) Zhang, E.; Zhang, S.; Wang, Q. Quantum Transport in a Curved One-Dimensional Quantum Wire with Spin-Orbit Interactions. Phys. Rev. B 2007, 75, 085308-1-08530810.

(24) Atanasov, V.; Dandoloff, R. Curvature-Induced Quantum Behaviour on a Helical Nanotube. Phys. Lett. A 2008, 372, 6141-6144.

(25) Ortix, C.; Kiravittaya, S.; Schmidt, O. G.; van den Brink, J. Curvature-Induced Geometric Potential in Strain-Driven Nanostructures. Phys. Rev. B 2011, 84, 045438-1$045438-5$.

(26) Entin, M. V.; Magarill, L. I. Spin-Orbit Interaction of Electrons on a Curved Surface. Phys. Rev. B 2001, 64, 085330-1-085330-5.

(27) da Costa, R. C. T. Quantum Mechanics of a Constrained Particle. Phys. Rev. A 1981, 23, 1982-1987.

(28) Nozaki, D.; da Rocha, C. G.; Pastawski, H. M.; Cuniberti, G. Disorder and Dephasing Effects on Electron Transport through Conjugated Molecular Wires in Molecular Junctions. Phys. Rev. B 2012, 8, 155327-1-155327-6.

(29) Mújica, V.; Kemp, M.; Ratner, M. A. Electron Conduction in Molecular Wires. II. Application to Scanning Tunneling Microscopy. J. Chem. Phys. 1994, 101, 6856-6864. 

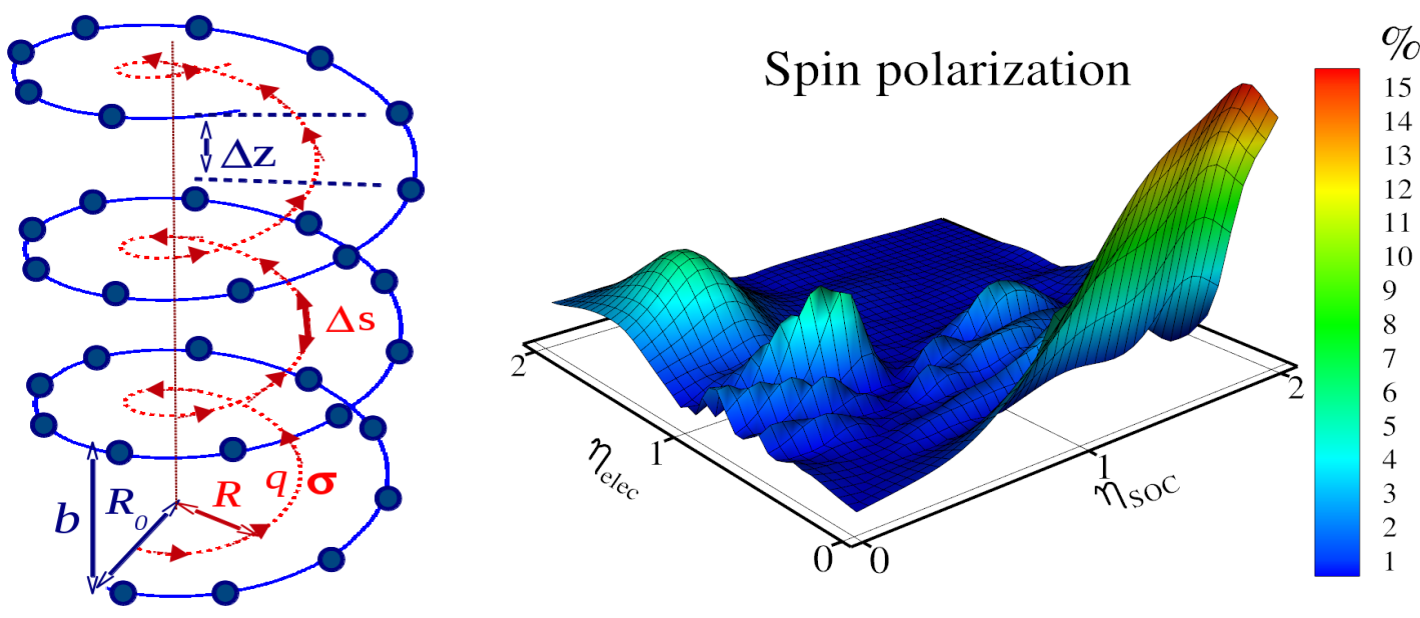

Figure 5: TOC 